\title{
Alteration of the Iron Level in Iron-overloaded Mice by Magnetospirillum Gryphiswaldense
}

Seyed Fazlollah Mousavi

Pasteur Institute of Iran

Seyed Davar Siadat

Pasteur Institute of Iran

\section{Tahereh Setayesh ( $\nabla$ tsetayesh@ucdavis.edu )}

University of California Davis department of pathology and laboratory medicine

Research

Keywords: Magnetospirillum gryphiswaldense, Iron Level Reduction, Iron Overload, Haemochromatosis

Posted Date: June 19th, 2020

DOI: https://doi.org/10.21203/rs.3.rs-36203/v1

License: (c) (1) This work is licensed under a Creative Commons Attribution 4.0 International License. Read Full License 


\section{Abstract}

\section{Background}

Magnetospirillum gryphiswaldense (MSR-1), a type of magnetotactic bacteria are capable of forming magnetosomes, which are nanometer-sized particles and membrane-enclosed organelles containing crystals of magnetite $\left(\mathrm{Fe}_{3} \mathrm{O}_{4}\right)$. For the synthesis of magnetosomes, bacteria require a significant amount of iron. Iron is an essential element for most life on earth; however, the elevation of iron level leads to hemochromatosis. This research aimed to investigate whether MSR-1 has an impact on the alteration of iron levels in mammalian cells in vivo.Female BALB/c were received intraperitonealinjections of irondextran (100mg/kg b.w.) 2 times per week for four consecutive weeks, and the iron was allowed to equilibrate for 15 days. The MSR-1 ( $10^{9}$ in $\left.200 \mu \mathrm{l} \mathrm{PBS}\right)$ were injected into healthy (C+MSR-1) and iron overloaded (IO+MSR-1) mice. The control healthy (C+ no MSR-1) and control iron overloaded (IO+ no MSR-1) mice received PBS. After the administration of bacteria, the viable bacterial numbers were evaluated in mice. Iron levels in serum and liver, as well as urinary iron exertion, were detected.

\section{Results}

Bacteria were not detected in the liver, spleen, and lymph nodes of mice after 72 hours of post-injection. Serum iron and total liver iron levels in C+MSR-1 were reduced compare to C-MSR-1 (by $42.1 \%$ and $34.1 \%)$, respectively. Moreover, similar patterns were observed in IO+MSR-1 compare to IO+ no MSR-1, $42.1 \%$ iron reduction in serum, and $32.1 \%$ in the liver after $24 \mathrm{~h}$ of post-injection. The urinary iron exertion was dramatically increased (by 3-fold) compared to groups with no MSR-1.

\section{Conclusion}

Our results suggest that MSR-1 can reduce the iron in iron-overloaded mice and, therefore, may inhibit the organ damage caused by excess iron. Our research on optimizing the biological magnetic system is continuing.

\section{Introduction}

Magnetotactic bacteria (MTB), such as the a-proteobacterium Magnetospirillum gryphiswaldense (MSR1), are motile and aquatic microorganisms that have a highly controlled bio-mineralization process to synthesize well-ordered nano-sized particles with perfect magnetic $\left(\mathrm{Fe}_{3} \mathrm{O}_{4}\right)$ and crystalline properties which allow the cells to align and to navigate along the geomagnetic field [1, 2]. MSR-1 accumulates iron (up to $4 \%$ of the dry weight) from the environment. It uses to biomineralize up to 60 magnetites $\left(\mathrm{Fe}_{3} \mathrm{O}_{4}\right)$ cuboctahedral crystals with a diameter of approximately 30 to 50 nanometers per cell $[1,3,4]$. These amounts indicate that MSR-1 has very efficient biological systems for uptake, transport, and precipitation of iron [3,5]. Based on Mössbauer spectroscopy and biochemical analyses, it was suggested the magnetite bio-mineralization pathway begins with iron uptake either as $\mathrm{Fe}^{2+}$ or $\mathrm{Fe}^{3+}$ from the environments, followed by a biochemical pool of iron composed of ferritin and $\mathrm{Fe}^{2+}$ within the cell $[3,6$, 
7]. In MSR-1, the iron uptake rate for $\mathrm{Fe}^{3+}$ is approximately 7-fold higher than that for $\mathrm{Fe}^{2+}$ at the same extracellular concentration [8].

Iron is a biologically important trace element which required for many metabolic pathways (i.e., DNA synthesis, cell growth, respiration) in living organisms [2, 9]; however, an increase of iron level in the body lead to iron-overloaded disorders such as hemochromatosis, is a disease in which too much iron builds up in the body [10]. Iron overload is an inevitable problem in patients because the body has no active mechanism to excrete excess iron [11]. Extra iron can cause damage in several organs such as spleen, liver, heart, and lymph nodes [10]. However, studies have demonstrated that iron-chelating drugs reduce organ damage and improve life expectancy in these patients [12]. In this study, the potential uptake of iron in mice by MSR-1 was investigated.

\section{Materials And Methods 2.1. Animals}

Female BALB/c mice (18-22 g, 4-6 weeks) were purchased from the laboratory animal center of Pasteur Institute of Iran. The Institutional Animal Care and Use Committee of Pasteur Institute of Iran (authorization no. 92/0201/3498) approved experiments involving mice. Mice were placed in polypropylene cages with stainless steel lids at room temperature with a 12-hour light/dark cycle. The animals had free access to standard pellet chow and drinking water, and body weights were measured weekly throughout the study period. The mice $(n=120)$ were divided into four groups, $i$. normal mice without MSR-1 ii. normal mice with MSR-1 iii. iron-overloaded mice with MSR-1 and iv. Iron-overloaded mice without MSR-1. For iron overloading, the mice received an intraperitoneal injection of $100 \mathrm{mg} / \mathrm{kg}$ iron dextran (Sigma-Aldrich) twice a week for 4-consecutive weeks, whereas the control group received PBS buffer. Two weeks after the last injection, iron was allowed to equilibrate in the mice [13-15].

\subsection{Bacterial strain and growth conditions}

Magnetospirillum gryphiswaldense MSR-1 (DSM6361) was purchased from Deutsche Sammlung von Mikroorganismen und Zellkulturen. These bacteria were cultured at $28^{\circ} \mathrm{C}$ using a modified Magnetospirillum growth medium (MSGM) containing $500 \mu \mathrm{M}$ ferric citrate as described previously [1618].

\subsection{MSR-1 injection and bacterial clearance}

For bacterial injection, MSR-1 cells were washed twice in PBS, then resuspended in PBS to give a final concentration of $1 \times 10^{9}$ MSR- 1 cells in $200 \mu \mathrm{L}$ PBS buffer, which was then injected into the tail vein of the mice and as a control, PBS buffer was injected $[19,20]$.

To detect the bacterial clearance, animals were sacrificed 24, 48, 72, and 96 hours after the first MSR-1 injection. The spleen, liver, and lymph nodes were harvested, rinsed with sterile PBS, weighed, and 
homogenized. The samples were centrifuged (1,000 rpm, 5 minutes), and supernatants from each sample were diluted and plated in $5 \mathrm{ml}$ of MSGM with $0.7 \%$ agar and incubated at $28{ }^{\circ} \mathrm{C}$ for ten days $[20,21]$.

\subsection{Detection of the iron level in plasma and liver}

The blood samples were collected by cardiac puncture into a centrifuge tube ( 3 iron-overloaded mice per each time point) [22]. Blood collection was performed between 8 a.m. to 10 a.m, and serum was obtained following centrifugation at 2,000 rpm for 20 minutes. The serum iron level was determined by the standard kit (Pars Azmoon, Tehran, Iran) with $5 \mu \mathrm{g} / \mathrm{dl}$ sensitivity.

The total iron-level was detected by using a wet washing procedure in the liver. The wet weight of the harvested liver was obtained ( $100 \mathrm{mg}$ ), and each one was placed in $2 \mathrm{ml}$ glass vials. The livers were desiccated at $65^{\circ} \mathrm{C}$ for 24 hours, then $200 \mu \mathrm{L}$ of an acid mixture (chloric acid: nitric acid 2:1) was added. After drying for 5 to 6 hours at $182^{\circ} \mathrm{C}, 1 \mathrm{ml}$ of $3 \mathrm{M} \mathrm{HCl}$ was added and mixed. A $50 \mu$ l aliquot of the acidified sample was incubated for 30 minutes with $200 \mu$ of chromogen (1.6 mM bathophenanthroline, $2 \mathrm{M}$ sodium acetate, and $11.5 \mathrm{mM}$ thioglycolic acid). The absorbance of the samples and iron standards was performed at $600 \mathrm{~nm}$, measured in triplicate, and the mean values were used for calculating the total amounts of iron in the liver [23].

\subsection{Analyze of urinary iron extraction}

The animals were placed in individual metabolic cages 24 hours before starting the experiment, and during this time, urine was collected and used as an initial reference point for each mouse. After injecting MSR-1 into the iron-overloaded mice, their urine was collected every 24 hours for ten days and then analyzed by using a flame atomic absorption spectrophotometer (Perkin-Elmer Model A Analyst 300) [24].

\subsection{Statistical analyses}

All of the results were measured in a minimum of 3 mice per experimental point. Comparisons between groups were analyzed by t-student test. For all comparisons, results with $p$ values $<0.05$ were considered significant. Statistical analyses were performed using Graphpad Prism 5.0 (Graphpad Software, San Diego, CA).

\section{Results}

For the iron-overloaded mice, no death occurred during the administration of iron dextran, and no weight gain was observed in all groups $(2.5 \pm 0.5 \mathrm{~g})$. Figure 1 illustrates the alteration of iron levels in serum and liver after administration of iron dextran. Serum iron and total liver iron levels increased during the four weeks of iron dextran administration compared with control animals that received PBS (non-iron overloaded mice). The total liver iron levels remained constant for two weeks after the final injection of iron dextran in the IO group.

The number of colony-forming units (CFUs) recovered in the liver and spleen peaked 24 hours after the first injection and then decreased to nearly zero by 72 hours, and after by 96 hours, there were no CFUs of 
MSR-1. The lymph nodes had virtually no CFUs during the entire experiment (Fig. 2).

Figure 3a shows that serum iron levels were decreased in Cont.+MSR-1 compare to Cont.+ no MSR-1 (by 42.1\%), and a similar pattern was also observed in IO + MSR-1 versus IO + no MSR-1 after 24 hours postinjection. The serum iron levels in $10+$ MSR-1 continued to be significantly less than $10+$ no MSR-1 during the ten days. However, the serum iron level in Cont. +MSR-1 group returned to the normal level after 96 hours post-injection.

The same pattern was observed in the total liver iron concentrations after biopsy during the first three days post-MSR-1 injection compare to no MSR-1 group (Fig. 3b). Moreover, the total iron levels in IO + MSR-1 were continued to be lower until the 10th-day post-injection, although it did not reach statistical significance.

After MSR-1 injection, the urinary iron excretion was analyzed. The iron levels were slightly increased (between 0 to 24 hours) after MSR-1 injection; however, iron excretion level was significantly increased after 24 hours and continued until 96 hours after post-injection compared to groups with no MSR-1. The iron excretion levels in 10 and control mice that received MSR-1 were similar (Figure. 3c).

\section{Discussion}

In this preliminary study, we try to understand whether MTB can uptake and accumulate iron from the animal body. This investigation was essential to understand the behavior of bacteria toward iron in in vivo environment. MTB have a remarkable potential to accumulate a large amount of iron in different environments and may play a key role in the regulation of the iron cycle in sedimentary environments, in which this ability is based on magnetosomes [25]. Bacteria require large amounts of iron to synthesis their magnetosomes; they may accumulate up to 100 times more iron than non-magnetotactic heterotrophic bacteria [26, 27].

In this study, to obtain the iron overloaded animal model, iron dextran was administrated twice a week for four consecutive weeks, and the iron level was measured as it was described earlier in mice [23] and rats [33]. As it is known, initially, iron dextran is taken up by the reticuloendothelial system, but then equilibrates with the parenchymal system [28]. In an earlier study by Moon et al., the author suggested a chronic iron overloaded model by administration of iron dextran for 2-8 weeks and showed that mice were not dead and adjusted well up to the cumulative iron dose of $300 \mathrm{mg}$ and long term survival was confirmed [29].

To investigate whether bacteria can uptake the iron in in vivo, MSR-1 was injected to iron overloaded mice, and normal mice and alteration of the iron level in plasma and liver were measured and compared with iron overloaded mice and normal mice which received only PBS. In a study by Benoit et al., Magnetospirillum magneticum (AMB-1) were injected intrathecal (i.t.) and intravascular (i.v.) to mice in order to visualize implanted tumors with magnetic resonance imaging by bacteria [30]. The authors showed that bacteria were cleared completely from other organs after six days of post-injection and 
remained only in tumors [30]. Setayesh et al. injected MSR-1, either i.v., peritoneal (i.p.) and subcutaneous (s.c.), and the clearance of MSR-1 in healthy mice was investigated [20]; the authors showed that mice clear MSR-1 from their body with different routes of injection after 96 hours. In the same study, the serum iron level was measured to understand by which routs of injection; bacteria effect on iron level [20].

In this experiment, after i.v. injection of MSR-1 to mice, serum iron, and total liver iron levels were decreased. The reduction of iron levels were highly significant during the first three days $(p<0.05)$. Notably, the percentages of serum and total liver iron reduction were similar in $10+$ MSR- 1 and in Cont.+MSR-1, which may explain the amount of iron taken by the same number of bacteria $\left(10^{9}\right.$ in $200 \mu \mathrm{l}$ PBS) are a consistent amount. Moreover, the CFUs of MSR-1 after 96 hours were zero in the liver, spleen, and lymph nodes that may explain the sharp reduction of iron levels during the first three days after the entrance of bacteria into the animal body. Therefore, it may conclude that as long as there are alive MSR1 in mice (during the first three days), iron levels in plasma and liver were reduced. The urinary iron excretion was higher between 24 to 96 hours after MSR-1 injection, and in parallel, the number of bacteria in mice was at the highest level in 24 hours post-injection, and it gradually reduced until 96 hours postinjection which we could not detect bacteria in liver, spleen and lymph nodes (see Fig. 3).

In this study, we reported the ability of iron uptake by MSR-1 in in vivo. MSR-1 has the potential to help to design a better biological tool that can multiply the effectiveness of the treatment of iron overloaded disorders. Therefore, our preliminary study opens the question of whether the bacteria can be a better replacement for chemical iron chelation drugs. However, our knowledge concerning the physiological characteristics and mechanism of iron uptake by MSR-1 in in vivo remains fragmentary. There are more studies warranted to understand the mechanism of bacterial clearance and cytotoxicity and genotoxicity effects of bacteria as well as an immune response in in vivo model. We also recommend future studies on optimizing the biological magnetic systems.

\section{Conclusion}

We found that MSR-1 significantly reduce the iron level in iron overloaded mice and increase the urinary iron excretion.

\section{Declarations}

\section{Availability of data and materials}

All data generated or analysed during this study are included in this published article

\section{Acknowledgments}

This study was supported by the Pasteur INS of Iran. The authors thank Prof. Siegfried Knasmueller at the Medical University of Vienna, Austria, for the fruitful discussion of the manuscript. 
The authors declare that there is no conflict of interest regarding the publication of this article.

\section{Abbreviations}

\begin{tabular}{|ll|}
\hline MTB & Magnetotactic bacteria \\
\hline MSR-1 & Magnetospirillum gryphiswaldense \\
\hline C+ no MSR-1 & Control mice without MSR-1 \\
\hline C+MSR-1 & Control healthy mice with MSR-1 \\
\hline IO+MSR-1 & Control Iron overloaded mice with MSR-1 \\
\hline IO+ no MSR-1 & Iron overloaded mice without MSR-1 \\
\hline CFUs & Colony-forming units \\
\hline
\end{tabular}

\section{References}

1. Bazylinski DA, Frankel RB. Magnetosome formation in prokaryotes. Nat Rev Microbiol. 2004;2(3):217-30.

2. Blakemore R. Magnetotactic bacteria. Science. 1975;190(4212):377-9.

3. Faivre D, et al. Intracellular Magnetite Biomineralization in Bacteria Proceeds by a Distinct Pathway Involving Membrane-Bound Ferritin and an Iron (II) Species. Angew Chem Int Ed. 2007;46(44):84959.

4. Moisescu C, et al. Controlled biomineralization of magnetite ( $\mathrm{Fe} 3 \mathrm{O} 4)$ by Magnetospirillum gryphiswaldense. Mineral Mag. 2008;72(1):333-6.

5. Schüler D, Baeuerlein E. Dynamics of iron uptake and Fe304 biomineralization during aerobic and microaerobic growth of Magnetospirillum gryphiswaldense. J Bacteriol. 1998;180(1):159-62.

6. Faivre D, Schüler D. Magnetotactic bacteria and magnetosomes. Chem Rev. 2008;108(11):4875-98.

7. Faivre $D$, et al. Intracellular magnetite biomineralization in bacteria proceeds by a distinct pathway involving membrane-bound ferritin and an iron(II) species. Angew Chem Int Ed Engl. 2007;46(44):8495-9.

8. Zhang $C$, et al. Two bifunctional enzymes with ferric reduction ability play complementary roles during magnetosome synthesis in Magnetospirillum gryphiswaldense MSR-1. J Bacteriol. 2013;195(4):876-85.

9. Xia $\mathrm{M}$, et al. A novel ferric reductase purified from Magnetospirillum gryphiswaldense MSR-1. Current microbiology. 2007;55(1):71-5.

10. Siddique A, Kowdley K. Review article: the iron overload syndromes. Aliment Pharmacol Ther. 2012;35(8):876-93. 
11. Poggiali E, et al. An update on iron chelation therapy. Blood transfusion = Trasfusione del sangue. 2012;10(4):411-22.

12. Alexander J, Kowdley KV. Hereditary hemochromatosis: genetics, pathogenesis, and clinical management. Ann Hepatol. 2005;4(4):240-7.

13. Mostafavi S, et al., Evaluation of Liver Toxicity of 2-Methyl-3-Hydroxypyridin-4-one in Iron Overloaded Rats. Iranian Journal of Pharmaceutical Sciences, 2006.

14. Moon SN, et al. Establishment of secondary iron overloaded mouse model: evaluation of cardiac function and analysis according to iron concentration. Pediatr Cardiol. 2011;32(7):947-52.

15. Navidi-Shishaone M, et al. Co-administration of Silymarin and Deferoxamine Against Kidney, Liver and Heart Iron Deposition in Male Iron Overload Rat Model. International Journal of Preventive Medicine. 2014;5(1):110.

16. Zhang Y, et al. Semicontinuous culture of Magnetospirillum gryphiswaldense MSR-1 cells in an autofermentor by nutrient-balanced and isosmotic feeding strategies. Appl Environ Microbiol. 2011;77(17):5851-6.

17. Komeili A, et al. Magnetosome vesicles are present before magnetite formation, and MamA is required for their activation. Proc Natl Acad Sci USA. 2004;101(11):3839-44.

18. Schultheiss D, Schüler D. Development of a genetic system for Magnetospirillum gryphiswaldense. Archives of microbiology. 2003;179(2):89-94.

19. Benoit MR, et al. Visualizing implanted tumors in mice with magnetic resonance imaging using magnetotactic bacteria. Clin Cancer Res. 2009;15(16):5170-7.

20. Setayesh T, Mousavi SF, Siadat SD. Effect of Magnetospirillum gryphiswaldense on serum iron levels in mice. Iranian journal of microbiology. 2012;4(3):160.

21. Xiang $L$, et al. Purified and sterilized magnetosomes from Magnetospirillum gryphiswaldense MSR-1 were not toxic to mouse fibroblasts in vitro. Lett Appl Microbiol. 2007;45(1):75-81.

22. Parasuraman $S$, Raveendran R, Kesavan R. Blood sample collection in small laboratory animals. Journal of pharmacology pharmacotherapeutics. 2010;1(2):87.

23. Kwik-Uribe $\mathrm{CL}$, et al. Chronic marginal iron intakes during early development in mice result in persistent changes in dopamine metabolism and myelin composition. J Nutr. 2000;130(11):282130.

24. Toliyat T, Jorjani M, Khorasanirad Z. An extended-release formulation of desferrioxamine for subcutaneous administration. Drug Deliv. 2009;16(7):416-21.

25. Lefèvre CT, Bazylinski DA. Ecology, Diversity, and Evolution of Magnetotactic Bacteria. Microbiol Mol Biol Rev. 2013;77(3):497-526.

26. Araujo ACV, et al. Magnetotactic bacteria as potential sources of bioproducts. Marine drugs. 2015;13(1):389-430.

27. Schuler D, Baeuerlein E. Iron-limited growth and kinetics of iron uptake in Magnetospirillum gryphiswaldense. Arch Microbiol. 1996;166(5):301-7. 
28. Sergejew T, Forgiarini P, Schnebli HP. Chelator-induced iron excretion in iron-overloaded marmosets. Br J Haematol. 2000;110(4):985-92.

29. Moon SN, et al. Establishment of secondary iron overloaded mouse model: evaluation of cardiac function and analysis according to iron concentration. Pediatr Cardiol. 2011;32(7):947-52.

30. Benoit MR, et al. Visualizing implanted tumors in mice with magnetic resonance imaging using magnetotactic bacteria. Clin Cancer Res. 2009;15(16):5170-7.

\section{Figures}


a. Serum Iron Level

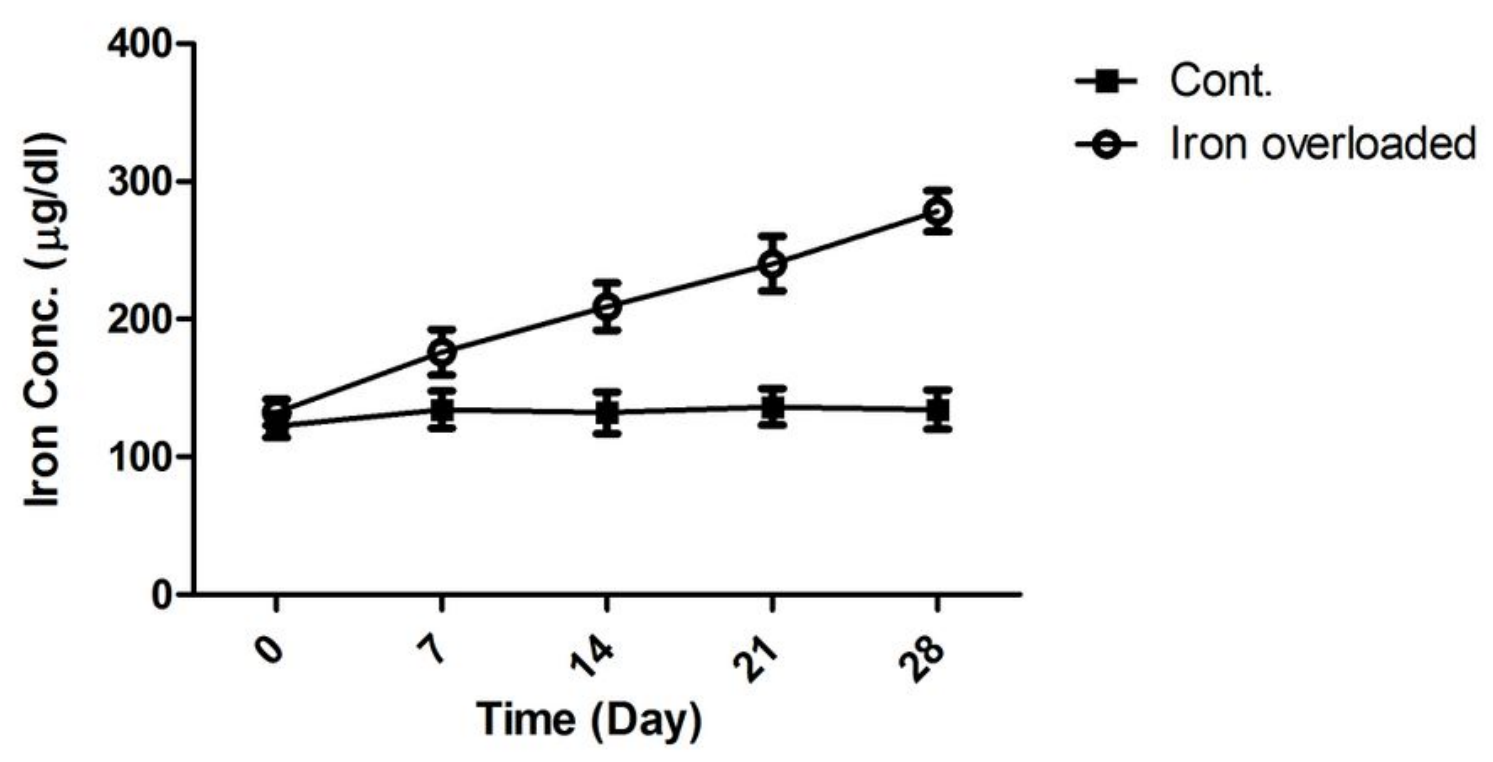

b. Liver Iron

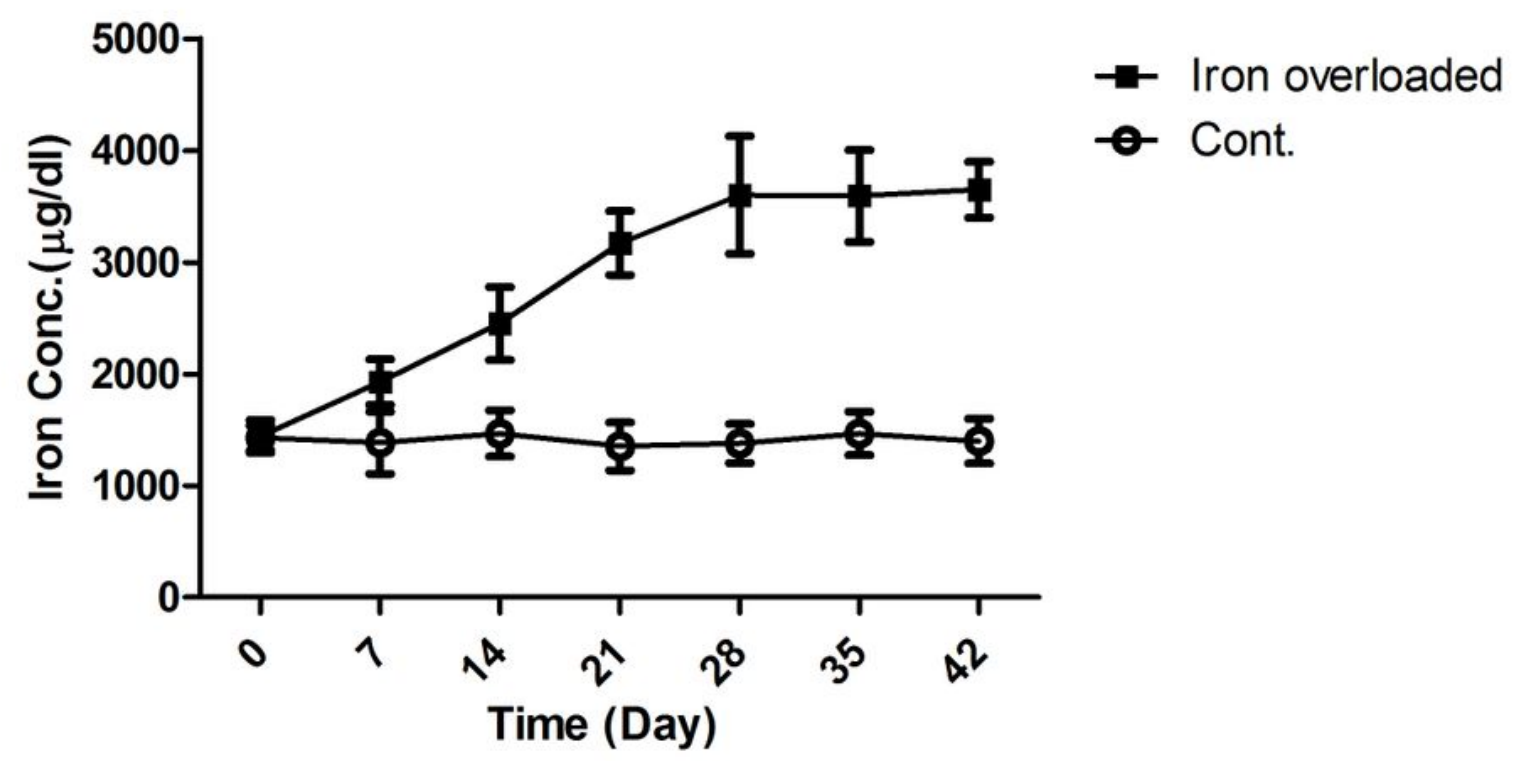

Figure 1

Changes in the serum iron level (a) and the liver total iron concentration (b) in mice during the iron overloading process. In iron overloaded mice, serum iron increased, and total liver iron increased during the 4 weeks of iron dextran administration and remained constant for two weeks after the final injection. 


\section{Colony Forming Unit (CFU)}

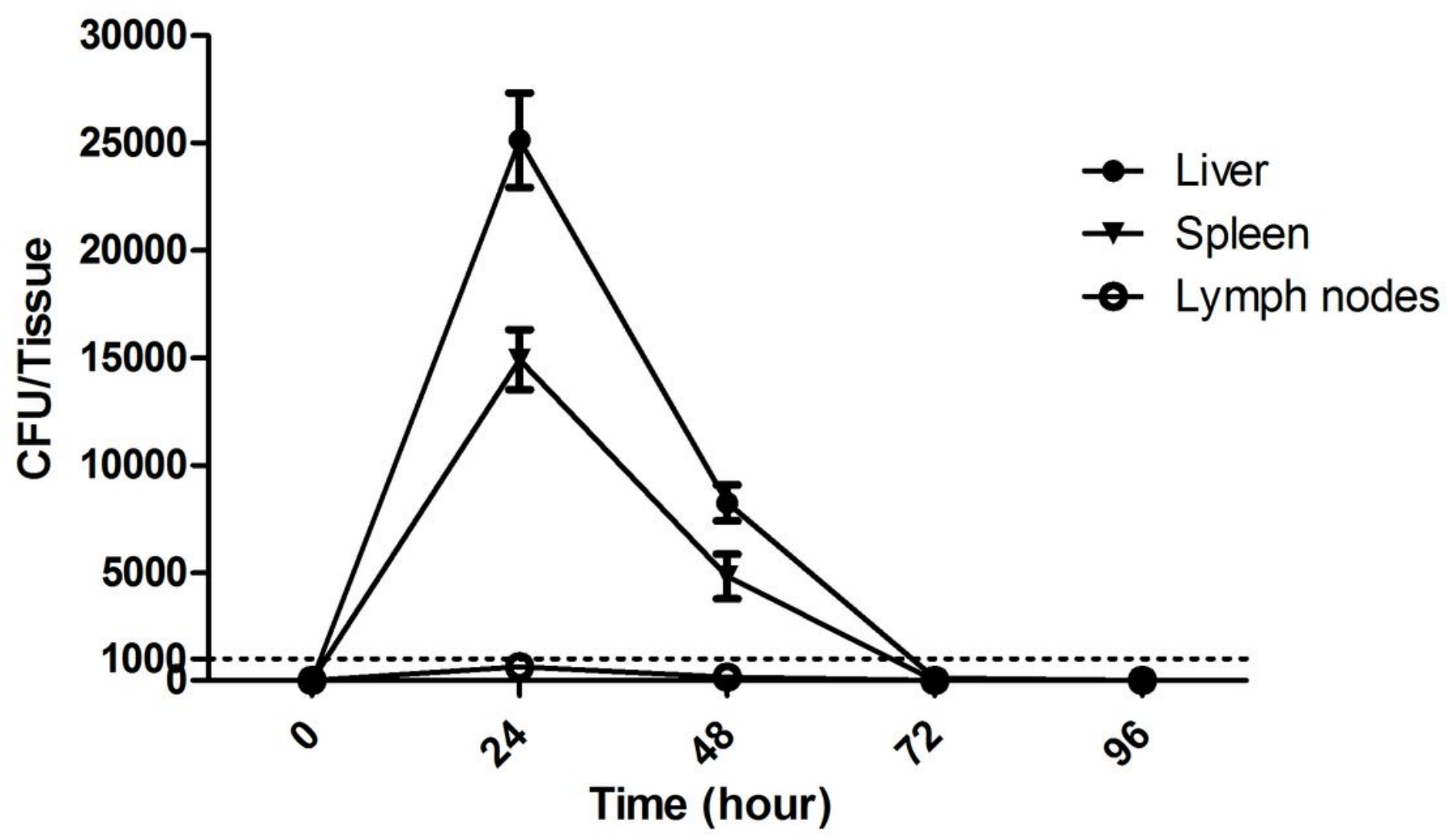

Figure 2

CFU per tissue after MSR-1 injection, the number of CFUs recovered in the liver and spleen peaked 24 hours after the first injection, then decreased to nearly zero by 72 hours, and by 96 hours, there were no CFUs. The lymph nodes had virtually no CFUs during the entire experiment. 
a.

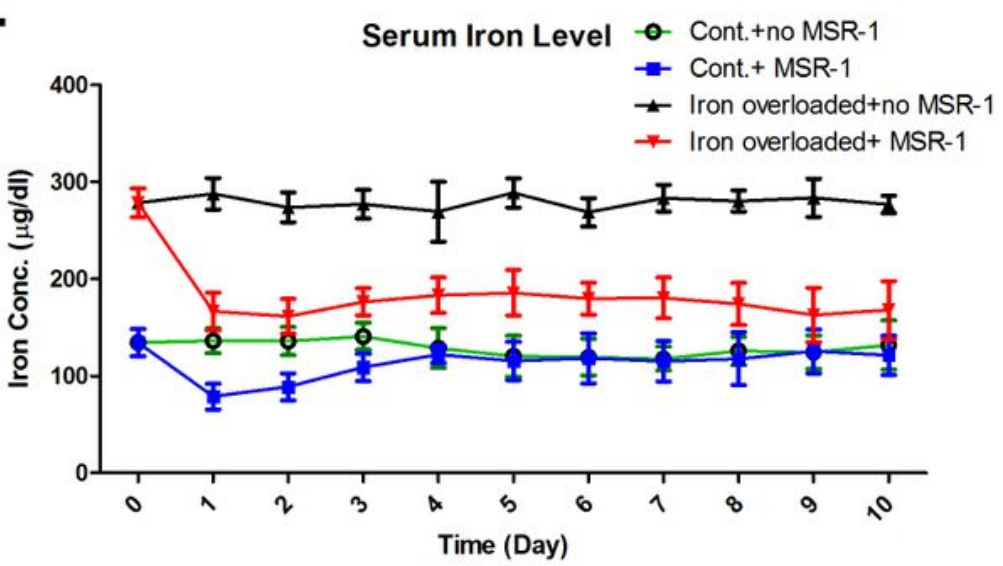

b.
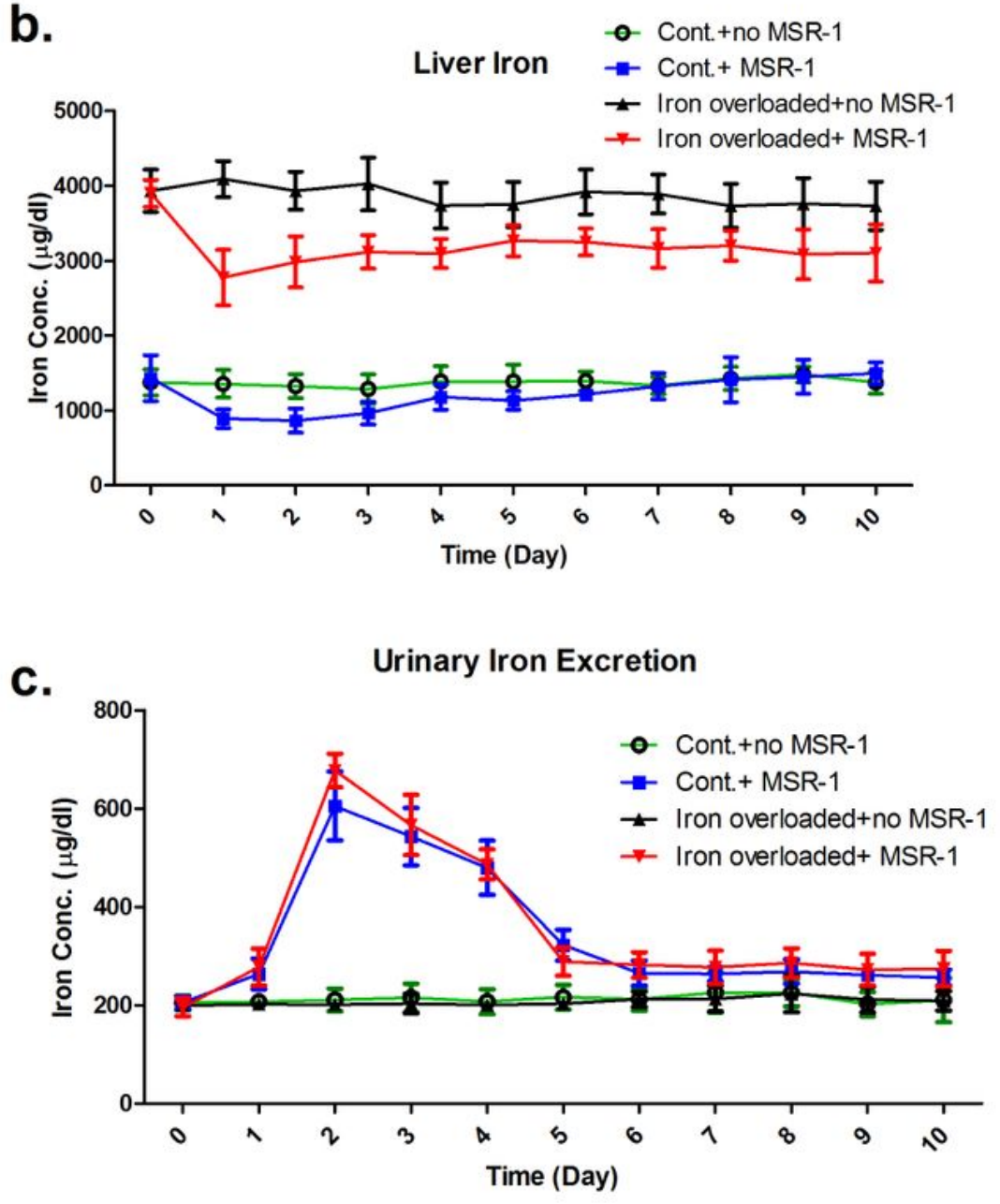

Figure 3

Serum (a) and total liver (b) iron levels after either MSR-1 or PBS (no MSR-1) injection in iron overloaded and control mice. Figure $3 c$ shows urinary iron excretion in iron overloaded and control mice after MSR-1 injection. 